\title{
Classical Solutions to the Initial-Boundary Value Problems for Nonautonomous Fractional Diffusion Equations
}

\author{
Jia Mu $\mathbb{D}^{1,2}$ Yang Liu, ${ }^{2}$ and Huanhuan Zhang $^{2}$ \\ ${ }^{1}$ Key Laboratory of Streaming Data Computing Technologies and Application, Northwest Minzu University, \\ Lanzhou 730000, China \\ ${ }^{2}$ School of Mathematics and Computer Science, Northwest Minzu University, Lanzhou 730000, China \\ Correspondence should be addressed to Jia Mu; mujia88@163.com
}

Received 14 August 2020; Revised 12 September 2020; Accepted 29 September 2020; Published 13 October 2020

Academic Editor: Yi-Sheng Lv

Copyright (c) 2020 Jia Mu et al. This is an open access article distributed under the Creative Commons Attribution License, which permits unrestricted use, distribution, and reproduction in any medium, provided the original work is properly cited.

\begin{abstract}
In this paper, we investigate a class of nonautonomous fractional diffusion equations (NFDEs). Firstly, under the condition of weighted Hölder continuity, the existence and two estimates of classical solutions are obtained by virtue of the properties of the probability density function and the evolution operator family. Secondly, it focuses on the continuity and an estimate of classical solutions in the sense of fractional power norm. The results generalize some existing results on classical solutions and provide theoretical support for the application of NFDE.
\end{abstract}

\section{Introduction}

Due to the nonlocal kernel of fractional differential operators, the time fractional diffusion equations (FDEs) of order 0 to 1 can describe irregular diffusion phenomena with long tails. In real life, the regular diffusion phenomenon (integer order case) only occurs in a few special cases. Therefore, FDE has attracted the attention of many scholars.

Qualitative analysis on FDE is the premise of practical application. At present, the research in this area mainly includes the existence, regularity, and stability of solutions. El-Sayed and Herzallah [1] discussed the maximal regularity of strong solutions of the autonomous fractional nonhomogeneous evolution equations under the condition of Hölder continuity. The existence and uniqueness of the mild solution of the autonomous fractional evolution equations (AFEE) involving almost sectorial operators and the existence of classical solutions under the condition of Hölder continuity were researched by Wang et al. [2]. Other studies on the maximal regularity of classical solutions in the autonomous case in the function space of Hölder continuous functions can refer to [3-5]. It is known that Hölder continuity is a special case of weighted Hölder continuity. Mu et al. [6] studied the existence, maximum regularity, and spatial regularity of classical solutions to the autonomous fractional diffusion equations (AFDE) under the condition of weighted Hölder continuity and extended some results in existing research. Later, the Mittag-Leffler function and eigenfunction expansion were employed by Zhou et al. [7] to study the existence, uniqueness, and regularity of mild solutions to the backward problem of the AFDE in the function space of weighted Hölder continuous functions. For other relevant results, please refer to [8-15].

The FDE are often nonautonomous in practical problems, which makes it necessary to research the NFDE. The diffusion coefficient of the NFDE is related not only to the spatial variable but also to the time variable, which brings great difficulties to the research. For example, the diffusion term generates a continuous semigroup in the autonomous case, rather than a two-parameter family of evolution operators in the nonautonomous case. Nevertheless, El-Borai [16] obtained the existence of classical solutions of non-autonomous fractional evolution equations (NFEE) under the condition of Hölder continuity. A new resolvent family concept and a fixed point theorem were used by Debbouche and Baleanu [17] to establish some control results for nonlocal impulsive quasilinear 
delay integrodifferential systems. Chalishajar et al. [18] used Sadovskii's fixed point theorem and Banach's fixed point theorem to study the existence of mild solutions to nonlocal problems of NFEE. In [19], the fractional resolvent family and the fixed point theorem are applied to investigate the global existence of mild solutions to NFEE. Chen et al. [20] applied noncompactness measure and Sadovskii's fixed point theorem to study the local existence and blow up of mild solutions to the Volterra-type NFEE. For other studies, see [21, 22]. On the basis of the above analysis, it can be found that the regularity of solutions to the NFDE and NFEE need to further study.

In this paper, we consider NFDE

$$
\left\{\begin{array}{l}
\partial_{t}^{\alpha} u(x, t)+\sum_{|p| \leq 2 m} b_{p}(x, t) D^{p} u(x, t)=f(x, t), \quad(x, t) \in \Omega \times I, \\
D^{p} u(x, t)=0, \quad|p| \leq m, \quad(x, t) \in \partial \Omega \times I \\
u(x, 0)=u_{0}(x), \quad x \in \Omega
\end{array}\right.
$$

where $\partial_{t}^{\alpha}$ is the Caputo fractional partial derivative with respect to $t, \Omega \subset R^{n}$ is a bounded open domain, whose boundary $\partial \Omega$ is sufficiently smooth, $I=(0, T) T>0, u_{0}$ is the initial data for $u$. $A(x, t)=\sum_{|p| \leq 2 m} b_{p}(x, t) D^{p} u(x, t), m$ is a positive integer, multi-index $x=\left(x_{1}, x_{2}, \ldots, x_{n}\right), p=\left(p_{1}\right.$, $\left.p_{2}, \ldots, p_{n}\right),|p|=\sum_{i=1}^{n} p_{i}, D^{p}=\left(\left(\partial^{p_{1}} / \partial x_{1}^{p_{1}}\right)\left(\partial^{p_{2}} / \partial x_{1}^{p_{2}}\right) \cdots(\partial\right.$ $\left.\left.p_{n} / \partial x_{1}^{p_{n}}\right)\right)$. Additionally, the following hypotheses are satisfied:

$\left(H_{0}\right)$ The operators $A(x, t)$ are uniformly elliptic operator in $\bar{\Omega} \times \bar{I}$. That is, there exists a constant $C$ such that

$$
C|\xi|^{2 m} \leq(-1)^{m} \operatorname{Re} \sum_{|p|=2 m} b_{p}(x, t) \xi^{p}, \quad(x, t) \in \bar{\Omega} \times \bar{I},
$$

where $\xi=\left(\xi_{1}, \xi_{2}, \ldots, \xi_{n}\right) \in \mathbb{R}^{n},|\xi|^{2}=\sum_{i=1}^{n} \xi_{i}^{2}$;

$\left(H_{1}\right)$ For $t \in \bar{I}$, the coefficients $b_{q}(x, t)$ are smooth functions with respect to $x \in \bar{\Omega}$, and there exists $0<\sigma \leq 1$ such that

$$
\left|b_{p}(x, t)-b_{p}(x, s)\right| \leq C|t-s|^{\sigma}, \quad \text { for } x \in \bar{\Omega} \text {, and } t, s \in \bar{I} .
$$

Firstly, when the inhomogeneous term satisfied the weighted Hölder continuity $f$ and the initial value $u_{0}$ belonged to $D(A(0))$, the recursive method is applied to determine the representation of the solution to (1). The existence of a unique classical solution to (1) is proved by virtue of the properties of the probability density function and the evolution operator family. In addition, some estimates of the classical solution, directly connected with the regularity of $u_{0}$ and $f$, are carried out. Finally, the continuity of the classical solution to (1) in some fractional power norm is proved and a reasonable estimate is obtained. Theorem 1 extends Theorem 2.2 in [16], where $f$ is Höler continuous.

The structure of this paper is as follows. The second section expounds the basic knowledge used later. In the third section, the existence and uniqueness of the classical solution to (1) and its continuity in the sense of fractional power are described, and the corresponding estimates are presented.

\section{Preliminaries}

Throughout this paper, the notation $C$ represents a constant in a particular situation, $\Gamma(\cdot)$ denotes the Gamma function, and $B(\cdot, \cdot)$ denotes the Beta function. Let $X$ be a Banach space with the norm $\|\cdot\|_{X}$ and $\gamma, \beta$ are two constants satisfying $0<\gamma<\beta \leq 1$. We define the space of weighted Hölder continuous functions, which is Hölder continuous with the exponent $\gamma$ and the weight $s^{1-\beta+\gamma}$, by $C^{\beta, \gamma}(I, X)=$ $\{h: \bar{I} \longrightarrow X \mid h$ is continuous on $I$ for $\beta \in(0,1)$ (or $\bar{I}$ for $\beta=1), \lim _{t \rightarrow 0} t^{1-\beta} f(t)$ exsits for $\beta \in(0,1)$,

$$
\begin{array}{r}
\sup _{0 \leq s<t \leq T} \frac{s^{1-\beta+\gamma}\|h(t)-h(s)\|_{X}}{(t-s)^{\gamma}}<\infty, \\
\left.\lim _{t \longrightarrow 0} \sup _{0 \leq s<t} \frac{s^{1-\beta+\gamma}\|h(t)-h(s)\|_{X}}{(t-s)^{\gamma}} \longrightarrow\right\} .
\end{array}
$$

The norm is

$$
\|h\|_{C^{\beta, \gamma}}=\sup _{0 \leq t \leq T} t^{1-\beta}\|h(t)\|_{X}+\sup _{0 \leq s<t \leq T} \frac{s^{1-\beta+\gamma}\|h(t)-h(s)\|_{X}}{(t-s)^{\gamma}} .
$$

Remark 1. If $h$ is Hölder continuous with exponent $\gamma(\gamma \in 0,1])$ on $\bar{I}$, then $h \in C^{1, \gamma}(I, X)[23]$. Because of this, our results could generalize some existing conclusions which need Hölder continuity.

Set $u(t)(x)=u(x, t), \quad f(t)(x)=f(x, t)$. Define $A(t): D \subset L^{2}(\Omega) \longrightarrow L^{2}(\Omega)$ by

$$
\begin{aligned}
A(t) u(t)(x) & =A(x, t) u(x, t), \\
D(A(t)) & =D=H^{2 m}(\Omega) \cap H_{0}^{m}(\Omega) .
\end{aligned}
$$

Then we turn (1) into the abstract fractional equations

$$
\left\{\begin{array}{l}
D_{t}^{\alpha} u(t)+A(t) u(t)=f(t), \quad t \in I \\
u(0)=u_{0}
\end{array}\right.
$$

in the Hilbert space $L^{2}(\Omega)$, where $D_{t}^{\alpha}$ is the Caputo fractional derivative. We say that $u: \bar{I} \longrightarrow L^{2}(\Omega)$ is a classical solution of (7), if $u(t)$ is continuous on $\bar{I}, A(t) u(t)$ and $D_{t}^{\alpha} u$ exist and are continuous on $I$, and (7) is satisfied on $\bar{I}$.

It is well known that each $-A(s)(s \in \bar{I})$ generates an analytic evolution family $\{T(t, s)\}_{t \geq 0}$. Under the assumptions $\left(H_{0}\right)$ and $\left(H_{1}\right)$, there exists a constant $k \geq 0$ such that $B(t):=A(t)+k I$ satisfies the following properties [24]:

$\left(H_{2}\right)$ For all $\lambda$ satisfying $\operatorname{Re} \lambda \leq 0$, the resolvent $R(\lambda ; B(t))$ of $B(t)$ exists and

$$
\|R(\lambda ; B(t))\|_{L^{2}(\Omega)} \leq \frac{C}{|\lambda|+1},
$$

for each $t \in \bar{I}$.

$\left(\mathrm{H}_{3}\right)$

$$
\left\|(B(t)-B(s)) B^{-1}(\tau)\right\|_{L^{2}(\Omega)} \leq C|t-s|^{\sigma},
$$

for all $t, s, \tau \in \bar{I}$. 
Without losing generality, we suppose $A(t)$ satisfies $\left(\mathrm{H}_{2}\right)$ and $\left(\mathrm{H}_{3}\right)$ in the following sections.

Set $U(t, s)=\alpha \int_{0}^{\infty} \theta \zeta_{\alpha}(\theta) T\left(t^{\alpha} \theta, s\right) \mathrm{d} \theta, \varphi_{1}(t, s)=(t-s)^{\alpha-1}$ $[A(t)-A(s)] U(t-s, s), \varphi_{n+1}(t, s)=\int_{s}^{t} \varphi_{n}(t, \tau) \varphi_{1}(\tau, s) \mathrm{d} \tau, n \in$ $\mathbb{Z}^{+}, \varphi(t, s)=\sum_{n=1}^{\infty} \varphi_{n}(t, s), V(t)=-A^{s}(t) A^{-1}(0)-\int_{0}^{t} \varphi(t, s) A$ $(s) A^{-1}(0) \mathrm{d} s$, where $t, s \in \bar{I}, \zeta_{\alpha}$ is probability density function defined on $(0, \infty)$ :

$$
\begin{aligned}
& \zeta_{\alpha}(\theta)=\frac{1}{\alpha} \theta^{-1-(1 / \alpha)} \rho_{\alpha}\left(\theta^{(-1 / \alpha)}\right), \\
& \rho_{\alpha}(\theta)=\frac{1}{\pi} \sum_{n=0}^{\infty}(-1)^{n-1} \theta^{-\alpha n-1} \frac{\Gamma(n \alpha+1)}{n !} \sin (n \pi \alpha), \quad \theta \in(0, \infty),
\end{aligned}
$$

and $\int_{0}^{\infty} \theta^{\lambda} \zeta_{\alpha}(\theta) \mathrm{d} \theta=(\Gamma(\lambda+1) / \Gamma(\alpha \lambda+1))$ for $\lambda \in(-1, \infty)$ [25]. In order to obtain the main results, we need to recall the fractional powers of $A(t)$ [24]. Let us denote by

$$
A^{-q}(t)=\frac{1}{\Gamma(q)} \int_{0}^{\infty} s^{q-1} T(s, t) \mathrm{d} s, \quad \text { for } q>0, t \geq 0 .
$$

Then we define the fractional power of $A(t)$ by $A^{q}(t)=$ $\left(A^{-q}(t)\right)^{-1}$ for $q>0$, and $A^{0}(t)=I$. The following conclusions follow from some results of $[16,23,24,26]$.

\section{Lemma 1}

(i) $(t-s)^{\alpha-1} U(t-s, s)$ and $(t-s)^{\alpha-1} A(t) U(t-s, s)$ are uniformly continuous, where $t, s \in \bar{I}, t-s \geq \mathcal{E}$, and $\epsilon$ is an arbitrary positive number;

(ii) $\|A(t)(U(t-s, s)-U(t-s, t))\|_{L^{2}(\Omega)} \leq C(t-s)^{\sigma-\alpha}$ for $0 \leq s<t \leq T$;

(iii) $\left\|A^{q}(s) U(t, s)\right\|_{L^{2}(\Omega)} \leq\left(C / t^{\alpha q}\right)$ for $q \geq 0, t>0, s \in \bar{I}$;

(iv) $\left\|A^{q}(s) T(t, s)\right\|_{L^{2}(\Omega)} \leq\left(C / t^{q}\right)$ for $q \geq 0, t>0, s \in \bar{I}$;

(v) $\left\|\varphi_{n}(t, s)\right\|_{L^{2}(\Omega)} \leq\left(C^{n}(t-s)^{\sigma n-1} / \Gamma(\sigma n)\right),\|\varphi(t, s)\|_{L^{2}(\Omega)}$ $\leq C(t-s)^{\sigma-1},\|V(t)\|_{L^{2}(\Omega)} \leq C+C t^{\sigma}, \varphi(t, s)$ and $V(t)$ are uniformly continuous in $t, s$, and

$$
\begin{array}{r}
\|\varphi(t, \tau)-\varphi(s, \tau)\|_{L^{2}(\Omega)} \leq C(t-s)^{\sigma-\nu}(s-\tau)^{\nu-1}, \quad \text { for } 0 \leq \tau<s<t \leq T, \\
\|V(t)-V(s)\|_{L^{2}(\Omega)} \leq C(t-s)^{\sigma}+C(t-s)^{\sigma-\nu} s^{\nu}, \quad \text { for } 0 \leq s<t \leq T,
\end{array}
$$

where $v \in(0, \sigma)$,

(vi) $\left\|A^{q_{1}}(t) A^{-q_{2}}(s)\right\| \leq C$, wheret, $s \in \bar{I}, 0 \leq q_{1}<q_{2}$;

(vii) $\left\|A(t) A^{-1}(s)\right\| \leq C$, where,$s \in \bar{I}$;

(viii) $A^{q}(t)(t \geq 0)$ is a closed operator, whose domain $D\left(A^{q}(t)\right)$ is a Banach space;

(ix) $A^{q_{1}+q_{2}}(t)=A^{q_{1}}(t) A^{q_{2}}(t)$, for $q_{1}, q_{2} \in \mathbb{R}$;

(x) $D\left(A^{q_{1}}(t)\right) \subset D\left(A^{q_{2}}(t)\right)$, for $0<q_{2} \leq q_{1}$.

\section{Classical Solutions}

In the following, we state the main results.

Theorem 1. If $f \in C^{\beta, \gamma}\left(I, L^{2}(\Omega)\right), \quad u_{0} \in D(A(0))$, $0<\gamma<\beta \leq 1$, and $\alpha+\beta>1$, then (7) has a unique classical solution:

$$
\begin{aligned}
u(t)= & u_{0}+\int_{0}^{t}(t-s)^{\alpha-1} U(t-s, s) V(s) A(0) u_{0} \mathrm{~d} s \\
& +\int_{0}^{t}(t-s)^{\alpha-1} U(t-s, s) f(s) \mathrm{d} s \\
& +\int_{0}^{t} \int_{0}^{\tau}(t-s)^{\alpha-1} U(t-s, s) \varphi(s, \tau) f(\tau) \mathrm{d} \tau \mathrm{d} s, \quad \text { for } t \in \bar{I},
\end{aligned}
$$

$$
\begin{aligned}
& \|u(t)\|_{L^{2}(\Omega)} \leq C \max \left\{1, t^{\alpha+\sigma}\right\}\left\|u_{0}\right\|_{L^{2}(\Omega)} \\
& +C \max \left\{t^{\alpha+\beta-1}, t^{\alpha+\sigma+\beta-1}\right\}\|f\|_{C^{\beta, \gamma},}, \quad \text { for } t \in \bar{I}, \\
& \|A(t) u(t)\|_{L^{2}(\Omega)}+\left\|D_{t}^{\alpha} u(t)\right\|_{L^{2}(\Omega)} \\
& \leq C\left\|A(0) u_{0}\right\|_{L^{2}(\Omega)}+C \max \left\{t^{\beta-1}, t^{2 \sigma+\beta-1}\right\}\|f\|_{C^{\beta, \gamma},}, \quad \text { for } t \in I . \\
& \text { Proof. We set } \\
& u(t)=u_{0}+\int_{0}^{t}(t-s)^{\alpha-1} U(t-s, s) V(s) A(0) u_{0} \mathrm{~d} s \\
& +\int_{0}^{t}(t-s)^{\alpha-1} U(t-s, s) w(s) \mathrm{d} s .
\end{aligned}
$$

Substituting it into (7), by Theorem 2.2 in [16], we get

$$
\begin{aligned}
& D^{\alpha}\left(u_{0}+\int_{0}^{t}(t-s)^{\alpha-1} U(t-s, s) V(s) A(0) u_{0} \mathrm{~d} s\right) \\
& \quad+A(t)\left(u_{0}+\int_{0}^{t}(t-s)^{\alpha-1} U(t-s, s) V(s) A(0) u_{0} \mathrm{~d} s\right)=0 .
\end{aligned}
$$

Then formally using Lemma 1 of [16], we obtain that 


$$
\begin{aligned}
f(t)= & D^{\alpha} \int_{0}^{t}(t-s)^{\alpha-1} U(t-s, s) w(s) \mathrm{d} s \\
& +A(t) \int_{0}^{t}(t-s)^{\alpha-1} U(t-s, s) w(s) \mathrm{d} s \\
= & w(t)-\int_{0}^{t}(t-s)^{\alpha-1} A(s) U(t-s, s) w(s) \mathrm{d} s \\
& +A(t) \int_{0}^{t}(t-s)^{\alpha-1} U(t-s, s) w(s) \mathrm{d} s \\
= & w(t)+\int_{0}^{t} \varphi_{1}(t-s, s) w(s) \mathrm{d} s .
\end{aligned}
$$

Write $w_{0}(t)=f(t), \quad w_{n+1}=-\int_{0}^{t} \varphi_{1}(t, s) w_{n}(s) \mathrm{d} s$, and $w(t)=\sum_{n=0}^{\infty} w_{n}(t)$. According to the character of $\varphi_{n}$ and Fubini's theorem, we have

$$
w_{n}(t)=\int_{0}^{t} \varphi_{n}(t, s) f(s) \mathrm{d} s .
$$

In view of Lemma 1 , we obtain that

$$
\begin{aligned}
\|w(t)-w(s)\|_{L^{2}(\Omega)} \leq & \|f(t)-f(s)\|_{L^{2}(\Omega)} \\
& +\int_{0}^{s}\|\varphi(t, \tau)-\varphi(s, \tau)\|_{L^{2}(\Omega)}\|f(\tau)\|_{L^{2}(\Omega)} \mathrm{d} \tau \\
& +\int_{s}^{t}\|\varphi(t, \tau) f(\tau)\|_{L^{2}(\Omega)} \mathrm{d} \tau \\
\leq & s^{-1+\beta-\gamma}(t-s)^{\gamma}\|f\|_{C^{\beta, \gamma}}+C\|f\|_{C^{\beta, \gamma}} \int_{0}^{s}(t-s)^{\sigma-\gamma}(s-\tau)^{\nu-1} \tau^{\beta-1} \mathrm{~d} \tau \\
& +C\|f\|_{C^{\beta, \gamma}} \int_{s}^{t}(t-\tau)^{\sigma-1} \tau^{\beta-1} \mathrm{~d} \tau \\
\leq & s^{-1+\beta-\gamma}(t-s)^{\gamma}\|f\|_{C^{\beta, \gamma}}+\operatorname{CB}(\nu, \beta)(t-s)^{\sigma-\nu} s^{\nu+\beta-1}\|f\|_{C^{\beta, \gamma}} \\
& +\frac{C}{\sigma} s^{\beta-1}(t-s)^{\sigma}\|f\|_{C^{\beta, \gamma}},
\end{aligned}
$$

provided $0<s<t \leq T$. Using Lemma 1 , we deduce that $u$ is continuous on $I$. In addition, we conclude from Lemma 1 that

$$
\begin{aligned}
\|u(t)-u(0)\|_{L^{2}(\Omega)} \leq & C \int_{0}^{t}(t-s)^{\alpha-1}\left(1+s^{\sigma}\right) \mathrm{d} s\left\|u_{0}\right\|_{L^{2}(\Omega)}+\left(C \int_{0}^{t}(t-s)^{\alpha-1} s^{\beta-1} \mathrm{~d} s\right) \\
& \left.+\int_{0}^{t} \int_{0}^{s}(t-s)^{\alpha-1}(s-\tau)^{\sigma-1} \tau^{\beta-1} \mathrm{~d} \tau \mathrm{d} s\right)\|f\|_{C^{\beta, \gamma}} \\
\leq & C\left(\frac{t^{\alpha}}{\alpha}+\mathrm{CB}(\alpha, \sigma+1) t^{\alpha+\sigma}\right)\left\|u_{0}\right\|_{L^{2}(\Omega)} \\
& +C\left(B(\alpha, \beta) t^{\alpha+\beta-1}+B(\sigma, \beta) B(\alpha, \sigma+\beta) t^{\alpha+\sigma+\beta-1}\right)\|f\|_{C^{\beta, \gamma},}
\end{aligned}
$$


implies that $u$ is continuous at $t=0$. Next, we show that we conclude from Lemma 1 and (22) that $u(t) \in D$ for $t \in I$. In view of

$$
\begin{aligned}
\int_{0}^{t}(t-s)^{\alpha-1} A(t) U(t-s, s) V(s) A(0) u_{0} \mathrm{~d} s \\
=\int_{0}^{t}(t-s)^{\alpha-1} A(t) U(t-s, t)(V(s)-V(t)) A(0) u_{0} \mathrm{~d} s \\
\quad+\int_{0}^{t}(t-s)^{\alpha-1} A(t)(U(t-s, s)-U(t-s, t)) V(s) A(0) u_{0} \mathrm{~d} s \\
\quad-\int_{0}^{\infty} \zeta_{\alpha}(\theta) T\left(t^{\alpha} \theta, t\right) V(t) A(0) u_{0} d \theta+V(t) A(0) u_{0},
\end{aligned}
$$

$$
\begin{aligned}
& \left\|A(t)\left(u_{0}+\int_{0}^{t}(t-s)^{\alpha-1} U(t-s, s) V(s) A(0) u_{0} \mathrm{~d} s\right)\right\|_{L^{2}(\Omega)} \\
& \leq\left\|A(t) A^{-1}(0) A(0) u_{0}\right\|_{L^{2}(\Omega)}+C \int_{0}^{t}(t-s)^{-1}\left((t-s)^{\sigma}+(t-s)^{\sigma-\nu} s^{\nu}\right) \mathrm{d} s\left\|A(0) u_{0}\right\|_{L^{2}(\Omega)} \\
& \quad+C \int_{0}^{t}(t-s)^{\sigma-1}\left(1+s^{\sigma}\right) \mathrm{d} s\left\|A(0) u_{0}\right\|_{L^{2}(\Omega)}+C\left(1+t^{\sigma}\right)\left\|A(0) u_{0}\right\|_{L^{2}(\Omega)} \\
& \leq C\left\|A(0) u_{0}\right\|_{L^{2}(\Omega)}+C t^{\sigma}\left\|A(0) u_{0}\right\|_{L^{2}(\Omega)}+C t^{2 \sigma}\left\|A(0) u_{0}\right\|_{L^{2}(\Omega)}
\end{aligned}
$$

That is, $u_{0}+\int_{0}^{t}(t-s)^{\alpha-1} U(t-s, s) V(s) A(0) u_{0} \mathrm{~d} s \in D$ for $t \in I$. We also know that $A(t)\left(u_{0}+\int_{0}^{t}(t-s)^{\alpha-1} U(t-\right.$

$$
\begin{aligned}
\int_{0}^{t}( & (t-s)^{\alpha-1} A(t) U(t-s, s) w(s) \mathrm{d} s \\
= & \int_{0}^{t}(t-s)^{\alpha-1} A(t) U(t-s, t)(w(s)-w(t)) \mathrm{d} s \\
& \quad+\int_{0}^{t}(t-s)^{\alpha-1} A(t)(U(t-s, s)-U(t-s, t)) w(s) \mathrm{d} s \\
& \quad-\int_{0}^{\infty} \zeta_{\alpha}(\theta) T\left(t^{\alpha} \theta, t\right) w(t) d \theta+w(t) \\
:= & I_{1}(t)+I_{2}(t)+I_{3}(t)+I_{4}(t) .
\end{aligned}
$$

Thanks to (22) and

$$
\begin{array}{r}
\|w(t)\|_{L^{2}(\Omega)} \leq t^{\beta-1}\|f\|_{C^{\beta, \gamma}}+\int_{0}^{t}(t-s)^{\sigma-1} s^{\beta-1} \mathrm{~d} s\|f\|_{C^{\beta, \gamma}} \\
\leq\left(t^{\beta-1}+B(\beta, \sigma) t^{\sigma+\beta-1}\right)\|f\|_{C^{\beta, \gamma}},
\end{array}
$$

drawn from Lemma 1, we come to the conclusion that 


$$
\begin{aligned}
& \left\|\int_{0}^{t}(t-s)^{\alpha-1} A(t) U(t-s, s) w(s) \mathrm{d} s\right\|_{L^{2}(\Omega)} \\
& \leq C \int_{0}^{t}(t-s)^{-1}\left(s^{-1+\beta-\gamma}(t-s)^{\gamma}+(t-s)^{\sigma-\gamma} s^{\nu+\beta-1}+s^{\beta-1}(t-s)^{\sigma}\right) \mathrm{d} s\|f\|_{C^{\beta, \gamma}} \\
& \quad+C \int_{0}^{t}(t-s)^{\sigma-1}\left(s^{\beta-1}+s^{\sigma+\beta-1}\right) \mathrm{d} s\|f\|_{C^{\beta, \gamma}}+C\left(t^{\beta-1}+t^{\sigma+\beta-1}\right)\|f\|_{C^{\beta, \gamma}} \\
& \leq C\left(B(\gamma, \beta-\gamma) t^{\beta-1}+B(\sigma-\nu, v+\beta) t^{\sigma+\beta-1}+B(\sigma, \beta) t^{\sigma+\beta-1}\right) \\
& \left.\quad+t^{\beta-1}+t^{\sigma+\beta-1}+B(\sigma, \sigma+\beta) t^{2 \sigma+\beta-1}\right)\|f\|_{C^{\beta, \gamma}} .
\end{aligned}
$$

That is $\int_{0}^{t}(t-s)^{\alpha-1} U(t-s, s) w(s) \mathrm{d} s \in D$ for $t \in[\varepsilon, T]$ $(\forall \varepsilon \in I))$. Next, we show that $A \int_{0}^{t}(t-s)^{\alpha-1} U(t-s, s) w(s) \mathrm{d} s \in C\left(I, L^{2}(\Omega)\right)$. Moreover,

$$
\begin{aligned}
I_{1}(t)-I_{1}(s)= & \int_{s}^{t}(t-\tau)^{\alpha-1} A(t) U(t-\tau, t)(w(\tau)-w(t)) \mathrm{d} \tau \\
& +\int_{0}^{s}\left((t-\tau)^{\alpha-1} A(t) U(t-\tau, t)-(s-\tau)^{\alpha-1} A(s) U(s-\tau, s)\right) \\
& \cdot(w(\tau)-w(s)) \mathrm{d} \tau+\int_{0}^{s}(t-\tau)^{\alpha-1} A(t) U(t-\tau, t)(w(s)-w(t)) \mathrm{d} \tau \\
:= & J_{1}(t)+J_{2}(t)+J_{3}(t),
\end{aligned}
$$

and for $t>s,(22)$ and Lemma 1 imply that

$$
\begin{aligned}
\left\|J_{1}(t)\right\|_{L^{2}(\Omega)} & \leq C \int_{s}^{t}\left(\tau^{-1+\beta-\gamma}(t-\tau)^{\gamma-1}+(t-\tau)^{\sigma-\nu-1} \tau^{\nu+\beta-1}+\tau^{\beta-1}(t-\tau)^{\sigma-1}\right) \mathrm{d} \tau \\
& \leq C\left(\frac{s^{-1+\beta-\gamma}}{\gamma}(t-s)^{\gamma}+\frac{\max \left\{s^{\nu+\beta-1}, t^{\nu+\beta-1}\right\}}{\sigma-\nu}(t-s)^{\sigma-\nu}+\frac{s^{\beta-1}}{\sigma}(t-s)^{\sigma}\right) .
\end{aligned}
$$

Then $\lim _{t \longrightarrow s^{+}} J_{1}(t)=0 \quad(s \in[\varepsilon, T], \forall \varepsilon \in I)$ holds. Furthermore, using Lemma 1 we have

$$
\begin{aligned}
\left\|(t-\tau)^{\alpha-1} A(t) U(t-\tau, t)(w(\tau)-w(s))\right\|_{L^{2}(\Omega)} & \leq C(t-\tau)^{-1}\|w(\tau)-w(s)\|_{L^{2}(\Omega)} \\
& \leq C\left(\tau^{-1+\beta-\gamma}(s-\tau)^{\gamma-1}+(s-\tau)^{\sigma-\gamma-1} \tau^{\nu+\beta-1}+\tau^{\beta-1}(s-\tau)^{\sigma-1}\right) \in L^{1}(I, \mathbb{R}) .
\end{aligned}
$$

Thus $\lim _{t \longrightarrow s^{+}} J_{2}(t)=0 \quad(s \in[\varepsilon, T], \forall \varepsilon \in I) \quad$ could be immediately gotten by Lebesgue's dominated convergence theorem. Applying Lemma 1, we also find that 


$$
\begin{aligned}
\left\|J_{3}(t)\right\|_{L^{2}(\Omega)} & \leq\left\|\left(\int_{0}^{\infty} \zeta_{\alpha}(\theta) T\left((t-s)^{\alpha} \theta, t\right) \mathrm{d} \theta-\int_{0}^{\infty} \zeta_{\alpha}(\theta) T\left(t^{\alpha} \theta, t\right) \mathrm{d} \theta\right)(w(t)-w(s))\right\|_{L^{2}(\Omega)} \\
& \leq C\|w(t)-w(s)\|_{L^{2}(\Omega)} .
\end{aligned}
$$

Then $\lim _{t \longrightarrow s^{+}} J_{3}(t)=0 \quad(s \in[\varepsilon, T])$ follows from (22).

Next, we write

$$
\begin{aligned}
I_{2}(t)-I_{2}(s)= & \int_{s}^{t}(t-\tau)^{\alpha-1} A(t)(U(t-\tau, \tau)-U(t-\tau, t)) w(\tau) \mathrm{d} \tau \\
& +\int_{0}^{s}\left((t-\tau)^{\alpha-1} A(t)(U(t-\tau, \tau)-U(t-\tau, t))-(s-\tau)^{\alpha-1} A(s)(U(s-\tau, \tau)-U(s-\tau, s))\right) w(\tau) \mathrm{d} \tau=: K_{1}+K_{2}
\end{aligned}
$$

Then

$$
\begin{aligned}
\left\|K_{1}(t)\right\|_{L^{2}(\Omega)} & \leq C \int_{s}^{t}(t-\tau)^{\sigma-1}\left(\tau^{\beta-1}+\tau^{\sigma+\beta-1}\right) \mathrm{d} \tau \\
& \leq \frac{C}{\sigma}\left(s^{\beta-1}+\max \left\{s^{\sigma+\beta-1}, t^{\sigma+\beta-1}\right\}\right)(t-s)^{\sigma},
\end{aligned}
$$

derived by Lemma 1 and (27). That is, $\lim _{t \longrightarrow s^{+}} K_{1}(t)=0$ $(s \in[\varepsilon, T], \forall \varepsilon \in I)$. Owing to

$$
\begin{array}{r}
\left\|(s-\tau)^{\alpha-1} A(s)(U(s-\tau, \tau)-U(s-\tau, t)) w(\tau)\right\|_{L^{2}(\Omega)} \\
\leq C(s-\tau)^{\sigma-1}\left(\tau^{\beta-1}+\tau^{\sigma+\beta-1}\right) \in L^{1}(I, \mathbb{R}),
\end{array}
$$

$\lim _{t \longrightarrow s^{+}} K_{2}(t)=0(s \in[\varepsilon, T], \varepsilon$ is arbitrary and $\varepsilon \in I)$ could be obtained by Lebesgue's dominated convergence theorem. In addition, Lemma 1 and the formula (22) imply that $\lim _{t \longrightarrow s^{+}} I_{3}(t)=\lim _{t \longrightarrow s^{+}} I_{4}(t)=0 \quad(s \in[\varepsilon, T])$. When $t<s$, the above limits are similar. Then by (16) and the properties of $w(t)$, using arguments similar to the ones in Theorem 2.2 and Lemma 1 in [16] one can easily obtain that $D_{t}^{\alpha} u$ exists and is continuous on $I$, and $u$ satisfies (7). Therefore, one obtains $u$ is a classical solution of (7). It is also easily seen that (14) and (15) hold, by (23), (25), and (28).

Remark 2. Theorem 1 extends Theorem 2.2 in [16], where $f$ is Höler continuous.

Theorem 2. If $f \in C^{\beta, \gamma}\left(I, L^{2}(\Omega)\right), \quad u_{0} \in D(A(0))$, $0<\gamma<\beta \leq 1, \alpha+\beta>1$, and $0<\beta_{1}<1-(1-\beta / \alpha)$, then the classical solution to (7) has the property: $A^{\beta_{1}}(t) u(t)$ is continuous on $\bar{I}$, and

$$
\left\|A_{1}^{\beta}(t) u(t)\right\|_{L^{2}(\Omega)} \leq C\left\|D\left(A^{\beta}(0)\right) u_{0}\right\|_{L^{2}(\Omega)}+C\|f\|_{C^{\beta, \gamma} .}
$$

Proof. The existence of the classical solution could be gotten immediately from Theorem 1 . Since $D(A(0)) \subset D\left(A^{\beta}(0)\right)$, $u_{0} \in D\left(A^{\beta}(0)\right)$. In view of $A^{\beta_{1}}(t)=A^{\beta_{1}-1}(t) A(t)$ and $A^{\beta_{1}-1}(t)$ is a bounded linear operator for $t \in \bar{I}$, using Theorem 1 we may find $A^{\beta_{1}}(t) u(t)$ is continuous on $I$. If $\alpha+\beta>1$, then $0<\beta_{1}<1-(1-\beta / \alpha)<\beta$. Next we show that $A^{\beta_{1}}(t) u(t)$ is continuous at $t=0$. In fact, note that

$$
\begin{aligned}
A^{\beta_{1}}(t) u(t)-A^{\beta_{1}}(0) u_{0}= & \left(A^{\beta_{1}}(t) A^{-\beta}(0)-A^{\beta_{1}-\beta}(0)\right) A^{\beta}(0) u_{0} \\
& +\int_{0}^{t}(t-s)^{\alpha-1} A^{\mu}(s) U(t-s, s) V(s) A^{-\mu}(t) A^{\beta_{1}}(t) A^{1-\beta}(0) A^{\beta}(0) u_{0} \mathrm{~d} s \\
& +\int_{0}^{t}(t-s)^{\alpha-1} A^{\beta_{1}-1}(t) A(t) U(t-s, s) w(s) \mathrm{d} s \\
=: & P_{1}(t)+P_{2}(t)+P_{3}(t),
\end{aligned}
$$

where $1-\beta+\beta_{1}<\mu<1$.

It is clear that $\left(H_{1}\right)$ implies that $\lim _{t \longrightarrow 0} P_{1}(t)=0$. We now estimate, using Lemma 1 and (27), 


$$
\begin{aligned}
\left\|P_{2}(t)\right\|_{L^{2}(\Omega)} & \leq C \int_{0}^{t}(t-s)^{\alpha-\alpha \mu-1}\left(1+s^{\sigma}\right) \mathrm{d} s\left\|A^{\beta}(0) u_{0}\right\|_{L^{2}(\Omega)} \\
& =C\left(t^{\alpha(1-\mu)}+B(\alpha(1-\mu), \sigma+1) t^{\alpha(1-\mu)+\sigma}\right)\left\|A^{\beta}(0) u_{0}\right\|_{L^{2}(\Omega)}, \\
\left\|P_{3}(t)\right\|_{L^{2}(\Omega)} & \leq\left\|\int_{0}^{t}(t-s)^{\alpha-1} A^{\beta_{2}}(s) A^{-\beta_{2}}(s) A^{\beta_{1}}(t) U(t-s, s) w(s) \mathrm{d} s\right\| \\
& \leq C \int_{0}^{t}(t-s)^{\alpha-\alpha \beta_{2}-1}\left(s^{\beta-1}+B(\beta, \sigma) s^{\sigma+\beta-1}\right)\|f\|_{C^{\beta, \gamma}} \mathrm{d} s \\
& \leq C\left(B\left(\alpha-\alpha \beta_{2}, \beta\right) t^{\alpha-\alpha \beta_{2}+\beta-1}+B(\beta, \sigma) B\left(\alpha-\alpha \beta_{2}, \beta+\sigma\right) t^{\alpha-\alpha \beta_{2}+\beta+\sigma-1}\right)\|f\|_{C^{\beta, \gamma},}
\end{aligned}
$$

where $\quad \beta_{1}<\beta_{2}<1-(1-\beta / \alpha)$. These prove $\lim _{t \rightarrow 0} P_{2}(t)=\lim _{t \rightarrow 0} P_{3}(t)=0$. From the above, we see that $A^{\beta_{1}}(t) u(t)$ is continuous on $\bar{I}$ and (36) holds.

\section{Data Availability}

The data used to support the findings of this study are included within the article.

\section{Conflicts of Interest}

The author declares that there are no conflicts of interest.

\section{Acknowledgments}

This research was supported by Scientific Research Foundation of the Higher Education Institutions of Gansu Province (2019B-022), Fundamental Research Funds for the Central Universities (31920180047), Gansu Provincial FirstClass Discipline Program of Northwest Minzu University (11080305), and Innovation Team of Intelligent Computing and Dynamical System Analysis and Application of Northwest Minzu University.

\section{References}

[1] A. M. A. El-Sayed and M. A. E. Herzallah, "Continuation and maximal regularity of fractional-order evolution equation," Journal of Mathematical Analysis and Applications, vol. 296, no. 1, pp. 340-350, 2004.

[2] R.-N. Wang, D.-H. Chen, and T.-J. Xiao, "Abstract fractional Cauchy problems with almost sectorial operators," Journal of Differential Equations, vol. 252, no. 1, pp. 202-235, 2012.

[3] C. Li and M. Li, "Holder regularity for abstract fractional cauchy problems with order in $(0,1)$," Journal of Applied Mathematics and Physics, vol. 6, no. 1, pp. 310-319, 2018.

[4] L. Liu, Z. Fan, G. Li, and S. Piskarev, "Maximal regularity for fractional cauchy equation in hölder space and its approximation," Computational Methods in Applied Mathematics, vol. 19, no. 4, pp. 779-796, 2019.

[5] R. Ponce, "Hölder continuous solutions for fractional differential equations and maximal regularity," Journal of Differential Equations, vol. 255, no. 10, pp. 3284-3304, 2013.

[6] J. Mu, B. Ahmad, and S. Huang, "Existence and regularity of solutions to time-fractional diffusion equations," Computers \& Mathematics with Applications, vol. 73, no. 6, pp. 985-996, 2017.

[7] Y. Zhou, J. Wei He, B. Ahmad, and N. Huy Tuan, "Existence and regularity results of a backward problem for fractional diffusion equations," Mathematical Methods in the Applied Sciences, vol. 42, no. 18, pp. 6775-6790, 2019.

[8] X. Su and M. Li, "The regularity of fractional stochastic evolution equations in Hilbert space," Stochastic Analysis and Applications, vol. 36, no. 4, pp. 639-653, 2018.

[9] G. A. Zou, B. Wang, and Y. Zhou, "Existence and regularity of mild solutions to fractional stochastic evolution equations," Mathematical Modelling of Natural Phenomena, vol. 13, no. 1, 2018.

[10] Y. Sarol, F. Viens, and F. Viens, "Time regularity of the evolution solution to fractional stochastic heat equation," Discrete \& Continuous Dynamical Systems-B, vol. 6, no. 4, pp. 895-910, 2006.

[11] Z. Fan, "Existence and regularity of solutions for evolution equations with Riemann-Liouville fractional derivatives," Indagationes Mathematicae, vol. 25, no. 3, pp. 516-524, 2014.

[12] J. L. Vázquez, A. de Pablo, F. Quirós, and A. Rodríguez, "Classical solutions and higher regularity for nonlinear fractional diffusion equations," Journal of the European Mathematical Society, vol. 19, no. 7, pp. 1949-1975, 2017.

[13] Z. Ji, H. Lin, S. Cao, Q. Qi, and H. Ma, "The complexity in complete graphic characterizations of multiagent controllability," IEEE Transactions on Cybernetics, 2020.

[14] L. Mo and S. Guo, "Consensus of linear multi-agent systems with persistent disturbances via distributed output feedback," Journal of Systems Science and Complexity, vol. 32, no. 3, pp. 835-845, 2019.

[15] S. Liu, Z. Ji, and H. Ma, "Jordan form-based algebraic conditions for controllability of multiagent systems under directed graphs," Complexity, vol. 2020, Article ID 7685460, 18 pages, 2020.

[16] M. El-Borai, “The fundamental solutions for fractional evolution equations of parabolic type," International Journal of Stochastic Analysis, vol. 11, no. 1, pp. 29-43, 2004.

[17] A. Debbouche and D. Baleanu, "Controllability of fractional evolution nonlocal impulsive quasilinear delay integro-differential systems," Computers \& Mathematics with Applications, vol. 62, no. 3, pp. 1442-1450, 2011.

[18] D. Chalishajar, D. Raja, K. Karthikeyan, and P. Sundararajan, "Existence results for nonautonomous impulsive fractional evolution equations," Results in Nonlinear Analysis, vol. 1, no. 3, pp. 133-147, 2018.

[19] B. Zhu, L. Liu, and Y. Wu, "Local and global existence of mild solutions for a class of nonlinear fractional reaction-diffusion equations with delay," Applied Mathematics Letters, vol. 61, pp. 73-79, 2016.

[20] P. Chen, X. Zhang, X. Zhang, and Y. Li, “A blowup alternative result for fractional nonautonomous evolution equation of Volterra type," Communications on Pure \& Applied Analysis, vol. 17, no. 5, pp. 1975-1992, 2018. 
[21] M. Mahmoud, E. Khairia, and G. Eman, "On some fractional evolution equations," Computers \& Mathematics with Applications, vol. 59, no. 3, pp. 1352-1355, 2010.

[22] P. Chen, X. Zhang, and Y. Li, "Cauchy problem for fractional non-autonomous evolution equations," Banach Journal of Mathematical Analysis, vol. 14, no. 2, pp. 559-584, 2020.

[23] A. Yagi, Abstract Parabolic Evolution Equations and Their Applications, Springer Science \& Business Media, Berlin, Germany, 2009.

[24] A. Pazy, Semigroups of Linear Operators and Applications to Partial Differential Equations, Springer Science \& Business Media, Berlin, Germany, 1992.

[25] M. M. El-Borai, "Some probability densities and fundamental solutions of fractional evolution equations," Chaos, Solitons \& Fractals, vol. 14, no. 3, pp. 433-440, 2002.

[26] A. Friedman, Partial Differential Equations, Holt, Rinehat and Winston, New York, NY, USA, 1969. 